\title{
Effect of Remifentanil Combined Anesthesia on Cytokines and Oxidative Stress in Patients undergoing Laparoscopic Surgery for Colon Cancer
}

\author{
Suchun Ding, Hongzhong Ma, Gao Wang, Zhenyu Yu, Kezhong Li and Aijie Huang
}

\begin{abstract}
Objective: To investigate the effect of remifentanil combined anesthesia on serum cytokines and oxidative stress indices in patients undergoing laparoscopic surgery for colon cancer.

Study Design: Experimental study.

Place and Duration of Study: Department of Anesthesiology, Yuhuangding Hospital Affiliated to Qingdao University, Yantai, China, from May 2016 to March 2018.

Methodology: A total of 154 patients undergoing laparoscopic surgery for colon cancer were randomly divided into control group and observation group, with 77 cases in each group. Control group received fentanyl combined anesthesia, and observation group received remifentanil combined anesthesia. Levels of serum cytokines IL-8, IL-6, CRP, TNF- $\alpha$ and the levels of oxidative stress indices SOD, MDA, CAT, and GSH on the first day after operation were compared. Occurrence of adverse reactions during anesthesia recovery was observed and recorded in both groups.

Results: On the first day after surgery, levels of serum cytokines IL-8, IL-6, CRP, TNF- $\alpha$ and MDA in the observation group were lower than those in the control group (all $p<0.001$ ); levels of serum SOD, GSH, and CAT in the observation group were higher than those in the control group (all $p<0.001$ ). The frequency of adverse reactions such as nausea and vomiting, chills, restlessness, cough, and tachycardia in the observation group was lower than that in the control group ( $p=0.029,0.016,0.009,0.025$, and 0.003 , respectively).

Conclusion: Compared with fentanyl combined anesthesia, the remifentanil combined anesthesia can significantly reduce serum levels of cytokines IL-8, IL-6, CRP, TNF- $\alpha$ and oxidative stress level, and is, therefore, more secure for patients undergoing laparoscopic surgery for colon cancer.
\end{abstract}

Key Words: Colon cancer, Laparoscopic surgery, Remifentanil, Fentanyl, Cytokines, Oxidative stress.

\section{INTRODUCTION}

Colon cancer is a common malignancy, accounting for about $55 \%$ of the world's total malignancies. It has been increasing year by year, and patients are younger. Most patients choose to undergo surgical treatment. 1 However, open surgery injury, hemodynamic changes, and severe pain can easily lead to stress response, induce inflammatory response, and increase oxidative stress level; thereby affecting the therapeutic effect and prognosis of colon cancer patients undergoing open surgery. 2,3 Laparoscopic surgery is a minimally invasive technique that has emerged in recent years. It greatly reduces the interference in the physiological functions of surgical patients and the patients restore quickly after surgery. Although laparoscopic surgery is less traumatic to the patient, the intraoperative procedure can still lead to stress response, and that caused by anesthesia is the

Department of Anesthesiology, Yuhuangding Hospital Affiliated to Qingdao University, Yantai, 264000, China

Correspondence: Dr. Aijie Huang, Department of Anesthesiology, Yuhuangding Hospital Affiliated to Qingdao University,

Yantai, 264000, China

E-mail: sangmi78814@163.com

Received: May 16, 2018; Accepted: September 02, 2018 most intense throughout the procedure.4,5 The ideal laparoscopic surgery requires rapid induction of anesthesia, adequate sedation and analgesia, rapid recovery after discontinuation, no restlessness, no respiratory depression or residual drug effects. 6 Remifentanil is a $\mu$-opioid receptor agonist of the fentanyl category and is an anesthetic and analgesic commonly used in general anesthesia. 7,8

The objective of this study was to investigate the effect of remifentanil combined anesthesia on serum cytokines and oxidative stress indices in patients undergoing laparoscopic surgery for colon cancer, in order to find the ideal anesthesia method and drugs to improve the quality of laparoscopic colon cancer surgery.

\section{METHODOLOGY}

This experimental study was done in the Department of Anesthesiology, Yuhuangding Hospital Affiliated to Qingdao University, Yantai, China, from May 2016 to March 2018. Approval was taken from the Hospital's Institutional Review Board. A total of 154 patients undergoing laparoscopic colon cancer surgery were enrolled in this study. Inclusion criteria were patients who were diagnosed by colonoscopy and pathological examination, colon cancer TNM stage belonged to stage 
I-II, and anesthesia grade (ASA) I-II. Exclusion criteria were patients who already received some form of treatment, with multiple lesions; coagulopathy, endocrine system, immune system disease, chronic infection and other diseases, other sites of malignancy, abnormal heart, lung, liver, kidney function, high-sensitivity constitution, a history of drug or alcohol abuse, and mental and neurological disorders. The patients were randomly divided into a control group and an observation group, with 77 cases in each group.

All patients routinely fasted before surgery and monitored in the operating room with vital signs such as electrocardiogram, blood oxygen saturation, respiration, and blood pressure. Masks of $6 \mathrm{~L} /$ minute were given for oxygen; chronic vein catheterisation was opened, and hydroxyethyl starch was administered at a 1:1 volume ratio with 130/0.4 sodium chloride injection and lactate ringer's injection $10 \mathrm{~mL} /(\mathrm{Kg} / \mathrm{hour})$. It was intravenously infused until the end of surgery.

Anesthesia induction method: The control group was given intravenous injection of midazolam $0.1 \mathrm{mg} / \mathrm{Kg}$ + propofol $2 \mathrm{mg} / \mathrm{Kg}+$ fentanyl citrate $2.5 \mu \mathrm{g} / \mathrm{Kg}$. The observation group was given intravenous injection of midazolam $0.1 \mathrm{mg} / \mathrm{Kg}$ + propofol $2 \mathrm{mg} / \mathrm{Kg}+$ remifentanil hydrochloride $2.5 \mu \mathrm{g} / \mathrm{Kg}$. After successful induction of anesthesia, mechanical ventilation via tracheal intubation was performed.

Intraoperative anesthesia maintenance: the control group was given fentanyl citrate injection, and the effect site concentration was $0.3 \mu \mathrm{g} /(\mathrm{Kg} / \mathrm{hour})+$ propofol injection 2-4 $\mathrm{mg} /(\mathrm{Kg} / \mathrm{hour})$, with continuous vein infusion by micro pump. The observation group received remifentanil hydrochloride $0.2 \mu \mathrm{g} /(\mathrm{Kg} /$ minute $)+$ propofol injection 2-4 $\mathrm{mg} /(\mathrm{Kg} / \mathrm{hour})$ for injection, with continuous vein infusion by micro pump.

During the operation, the bispectral index (BIS) monitor was used to monitor the depth of anesthesia, adjust the amount of propofol, and maintain the BIS at 45-55. Intraoperative intermittent administration of $0.05 \mathrm{mg} / \mathrm{Kg}$ vecuronium bromide for injection was used to maintain muscle relaxation. All patients underwent laparoscopic radical colon cancer surgery by the same group of physicians. The propofol infusion was discontinued 30 minutes before the end of surgery, and fentanyl and remifentanil were infused until the end of surgery. After the patient's consciousness was restored, the endotracheal tube was removed and sent to the recovery room for resuscitation.

The levels of serum cytokines IL-8, IL-6, CRP, and TNF- $\alpha$ and the levels of oxidative stress indices SOD, MDA, CAT, and GSH on the first day after surgery were compared. Serum levels of IL-8, IL-6, CRP, and TNF- $\alpha$ were measured by enzyme-linked immunosorbent assay (ELISA). The SOD level was measured by xanthine oxidase method. Serum MDA was detected by thiobarbituric acid method. Serum CAT level was measured by spectrophotometry. Serum GSH level was detected by dithiodinitrobenzoic acid colorimetry. The occurrence of adverse reactions during anesthesia recovery was observed, recorded and compared between the two groups of patients.

SPSS 25.0 software is adopted for data statistical analysis. Measurement data shown in Mean $\pm S D$ was examined by independent sample t-test. Enumeration data was represented by $n(\%)$, and $X^{2}$ test is adopted to examine them. The $p<0.05$ was taken as statistical significance in the difference.

\section{RESULTS}

Among the 154 patients, there were $85(55.19 \%)$ males and $69(44.81 \%)$ females; aged $41-67$ years, with mean age of $56.35 \pm 4.81$ years; and weight from $50-75 \mathrm{~kg}$, mean being $62.15 \pm 7.43 \mathrm{~kg}$. The operation time ranged from 201-287 minutes, average being $237.61 \pm 36.52$

Table I: Comparison of serum cytokines levels on the first day after surgery.

\begin{tabular}{|c|c|c|c|c|c|c|c|c|c|}
\hline \multirow[t]{2}{*}{ Groups } & \multirow[t]{2}{*}{$\mathrm{n}$} & \multicolumn{2}{|c|}{ IL-8 (pg/mL) } & \multicolumn{2}{|c|}{ IL-6 (pg/mL) } & \multicolumn{2}{|c|}{ CRP (mg/L) } & \multicolumn{2}{|c|}{$\mathrm{TNF}-\alpha(\mathrm{pg} / \mathrm{mL})$} \\
\hline & & Mean \pm SD & $\mathrm{p}$-value & Mean \pm SD & $\mathrm{p}$-value & Mean \pm SD & $\mathrm{p}$-value & Mean \pm SD & $p$-value \\
\hline Control group & 77 & $69.65 \pm 4.71$ & $<0.001$ & $74.67 \pm 10.26$ & $<0.001$ & $19.73 \pm 8.52$ & $<0.001$ & $47.36 \pm 6.99$ & $<0.001$ \\
\hline Observation group & 77 & $50.24 \pm 3.45$ & & $52.32 \pm 6.79$ & & $11.62 \pm 2.37$ & & $35.23 \pm 6.20$ & \\
\hline
\end{tabular}

Table II: Comparison of serum oxidative stress markers on the first day after surgery.

\begin{tabular}{|c|c|c|c|c|c|c|c|c|c|}
\hline \multirow[t]{2}{*}{ Groups } & \multirow[t]{2}{*}{$\mathrm{n}$} & \multicolumn{2}{|c|}{$\mathrm{SOD}(\mathrm{nU} / \mathrm{mL})$} & \multicolumn{2}{|c|}{$\mathrm{MDA}(\mathrm{mmol} / \mathrm{L})$} & \multicolumn{2}{|c|}{ CAT (U/mL) } & \multicolumn{2}{|c|}{$\mathrm{GSH}(\mathrm{mg} / \mathrm{mL})$} \\
\hline & & Mean \pm SD & $p$-value & Mean \pm SD & $p$-value & Mean \pm SD & $p$-value & Mean \pm SD & $\mathrm{p}$-value \\
\hline Control group & 77 & $75.83 \pm 5.64$ & $<0.001$ & $7.87 \pm 1.03$ & $<0.001$ & $80.65 \pm 6.17$ & $<0.001$ & $60.54 \pm 8.21$ & $<0.001$ \\
\hline Observation group & 77 & $80.16 \pm 8.43$ & & $5.35 \pm 0.55$ & & $89.83 \pm 9.03$ & & $70.16 \pm 12.57$ & \\
\hline
\end{tabular}

Table III: Comparison of adverse reactions during anesthesia recovery.

\begin{tabular}{l|c|c|c|c|c|c|c|c|c|c|c}
\hline Groups & \multirow{2}{*}{$\mathrm{n}$} & \multicolumn{2}{|c|}{ Nausea and vomiting } & \multicolumn{2}{|c|}{ Chills } & \multicolumn{2}{c|}{ Restlessness } & \multicolumn{2}{|c}{ Cough } & \multicolumn{2}{c}{ Tachycardia } \\
\cline { 3 - 10 } & & $\mathrm{n}(\%)$ & $\mathrm{p}$-value & $\mathrm{n}(\%)$ & $\mathrm{p}$-value & $\mathrm{n}(\%)$ & $\mathrm{p}$-value & $\mathrm{n}(\%)$ & $\mathrm{p}$-value & $\mathrm{n}(\%)$ & $\mathrm{p}$-value \\
\hline Control group & 77 & $9(11.69)$ & 0.029 & $8(10.39)$ & 0.016 & $9(11.69)$ & 0.009 & $11(14.29)$ & 0.025 & $11(14.29)$ & 0.003 \\
Observation group & 77 & $2(2.60)$ & & $1(1.30)$ & & $1(1.30)$ & & $3(3.90)$ & & $1(1.30)$ & \\
\hline
\end{tabular}


minutes. TNM stage was stage I in $62(40.26 \%)$ and stage II in 92 (59.74\%).

On the first day after surgery, levels of serum cytokines IL-8, IL-6, CRP and TNF- $\alpha$ were lower in the observation group than those in the control group (all $p<0.001$, Table I). On the first day after surgery, serum MDA level was lower in the observation group than that in the control group ( $p<0.001$ ); levels of serum SOD, GSH, and CAT in the observation group were higher than those in the control group (all $p<0.001$, Table II). The frequency of adverse reactions such as nausea and vomiting, chills, restlessness, cough, and tachycardia was lower in the observation group than that in the control group $(p=0.029,0.016,0.009,0.025$ and 0.003 , respectively, Table III).

\section{DISCUSSION}

Surgical stress response is a major factor in the occurrence of complications such as stress-induced gastrointestinal ulcers and hemorrhage, which seriously affect the surgical outcome and the patient's prognosis. Anesthetic agents play an important role in the regulation of stress response. Selecting appropriate anesthetic drugs can prevent or reduce stress response and complications.9,10 Remifentanil is a potent anesthetic. With rapid onset and rapid elimination, its analgesic effect is far stronger than fentanyl. It is not easy to accumulate, and can reduce the secretion of cortisol, effectively inhibiting pneumoperitoneum and surgically induced cardiovascular responses. Now, it is commonly used for induction of general anesthesia. ${ }^{11,12}$ Propofol is a short-acting intravenous anesthetic that is characteristic of rapid onset, short half-life, strong controllability, no accumulation in the body and low toxicity. ${ }^{13,14}$ The combination of remifentanil and propofol has a synergistic effect, which can reduce the dose of propofol to $75 \%$, and propofol has no effect on the clearance rate of remifentanil. 15,16 This study showed that the incidence of adverse reactions such as nausea and vomiting, chills, restlessness, cough, and tachycardia was lower in the observation group than that in the control group. It suggested that the safety of remifentanil combined anesthesia is higher than that of fentanyl combined anesthesia.

IL-6 is a pro-inflammatory cytokine, the level of which is positively correlated with the degree of immune system damage. It can be used to evaluate the prognosis of colon cancer. ${ }^{17}$ The biological effects of IL-8 and CRP are quite strong, of which CRP is considered as a higher sensitivity indicator of increased tissue damage.18 TNF- $\alpha$ is derived from activated macrophages and can induce inflammatory responses and promote the synthesis of multiple cytokines. 19 The results of this study showed that serum levels of IL-8, IL-6, CRP and TNF- $\alpha$ were lower in the observation group than those in the control group on the first day after surgery. It suggested that remifentanil combined anesthesia can reduce the level of postoperative inflammatory markers in patients. The result is basically consistent with the studies of other literatures on this issue. 20

Oxidative stress is a stress response caused by reactive oxygen species, and it can cause tissue peroxidative damage. During the laparoscopic surgery, the artificial pneumoperitoneum needs to be established and removed; the intra-abdominal pressure is changed; ischemia-reperfusion is prone to occur, leading to oxidation-anti-oxidation system dysfunction; therefore, oxidative stress increases. ${ }^{21}$ In the oxidative stress indices, MDA can reflect the level of oxygen-free radicals in tissues and the degree of tissue damage; CAT, SOD, and GSH are all antioxidant enzymes, which can reduce the harm caused by oxidative stress. 22 SOD can clear the superoxide radical of the body. CAT can catalyse the decomposition of hydrogen peroxide into oxygen and water. GSH has the physiological functions of scavenging free radicals, anti-oxidation and antiaging. Increased MDA and decreased CAT, SOD, and GSH can easily lead to oxidative stress injury. The results of this study showed that serum MDA levels are lower in the observation group than those in the control group on the first day after surgery. Serum SOD, GSH, and CAT levels are higher in the observation group than those in the control group. It suggested that remifentanil combined anesthesia can significantly reduce postoperative oxidative stress.

\section{CONCLUSION}

Compared with fentanyl combined anesthesia, the remifentanil combined anesthesia can significantly reduce serum levels of cytokines IL-8, IL-6, CRP, and TNF- $\alpha$ in elderly patients undergoing laparoscopic colon cancer surgery and oxidative stress level; and is, therefore, more secure.

\section{REFERENCES}

1. Bhurgri H, Samiullah S. Colon cancer screening - Is it time yet? J Coll Physicians Surg Pak 2017; 27:327-8.

2. Martínezpérez A, Brunetti F, Vitali GC, Abdalla S, Ris F, De'Angelis N. Surgical treatment of colon cancer of the splenic flexure: A systematic review and meta-analysis. Surg Laparosc Endosc Percutan Tech 2017; 27:318-27.

3. Salem JF, Gummadi S, Marks JH. Minimally invasive surgical approaches to colon cancer. Surg Oncol Clin N Am 2018; 27:303-18.

4. Ece I, Ozturk B, Yilmaz H, Yormaz S, Sahin M. The effect of single incision laparoscopic cholecystectomy on systemic oxidative stress: a prospective clinical trial. Ann Surg Treat Res 2017; 92:179-83.

5. Buunen MR, Veldkamp R, Hop W, Kuhry E, Jeekel J, Haglind E, et al. Survival after laparoscopic surgery versus open surgery 
for colon cancer: long-term outcome of a randomised clinical trial. Lancet Oncology 2009; 10:44-52.

6. Bajwa SJ, Kulshrestha A. Anaesthesia for laparoscopic surgery: general vs regional anaesthesia. J Minim Access Surg 2016; 12:4-9.

7. Cappuccio E, Thung AK, Tobias JD. General anesthesia with dexmedetomidine and remifentanil in a neonate during oracotomy and resection of a congenital cystic adenomatoid malformation. J Pediatr Pharmacol Ther 2018; 23:215-8.

8. Kim HY, Lee SY, Kang S, Kim B, Moon YR, Kim JE. Effects of age on effect-site concentration of remifentanil for suppressing anesthetic emergence cough in male patients undergoing laparoscopic cholecystectomy. Clin Interv Aging 2018; 13: 1053-60.

9. Dang Y, Shi X, Xu W, Zuo M. The effect of anesthesia on the immune system in colorectal cancer patients. Can $J$ Gastroenterol Hepatol 2018; 2018:7940603.

10. Alirezaei M, Rezaei M, Hajighahramani S, Sookhtehzari A, Kiani K. Oleuropein attenuates cognitive dysfunction and oxidative stress induced by some anesthetic drugs in the hippocampal area of rats. J Physiol Sci 2016; 67:1-9.

11. Palumbo P, Usai $S$, Amatucci $C$, Perotti $B$, Ruggeri L, Illuminati $G$, et al. Inguinal hernia repair in day surgery: The role of MAC (monitored anesthesia care) with remifentanil. G Chir 2017; 38: 273-9.

12. Zhang Y, Li Y, Wang H, Cai F, Shen S, Luo X. Correlation of MDR1 gene polymorphism with propofol combined with remifentanil anesthesia in pediatric tonsillectomy. Oncotarget 2017; 9:20294-303.

13. Bang YS, Kim YU, Oh D, Shin EY, Park SK. A randomized, double-blind trial evaluating the efficacy of palonosetron with total intravenous anesthesia using propofol and remifentanil for the prevention of postoperative nausea and vomiting after gynecologic surgery. J Anesth 2016; 30:935-40.
14. Zheng X, Cong J, Zhang H, Chu X. Personalized analysis of pathway aberrance induced by sevoflurane and propofol. Mol Med Rep 2017; 16:5312-20.

15. Rocha RG, Almeida EG, Carneiro LMM, Almeida NF, Boas WWV, Gomez RS. Anesthesia recovery comparison between remifentanil-propofol and remifentanil-desflurane guided by Bispectral Index monitoring. Rev Bras Anestesiol 2017; 67:500-7.

16. Shin S,Kim S. Dental treatment in patients with severe gag reflex using propofol-remifentanil intravenous sedation. J Dent Anesth Pain Med 2017; 17:65-9.

17. Guthrie GJ, Roxburgh CS, Richards CH, Horgan PG, McMillan DC. Circulating IL-6 concentrations link tumour necrosis and systemic and local inflammatory responses in patients undergoing resection for colorectal cancer. $\mathrm{Br} J$ Cancer 2013; 109:131-7.

18. Vainer N, Dehlendorff C, Johansen JS. Systematic literature review of IL-6 as a biomarker or treatment target in patients with gastric, bile duct, pancreatic and colorectal cancer. Oncotarget 2018; 9:29820-41.

19. Godos J, Biondi A, Galvano F, Basile F, Sciacca S, Giovannucci EL, et al. Markers of systemic inflammation and colorectal adenoma risk: Meta-analysis of observational studies. World J Gastroenterol 2017; 23:1909-19.

20. Qi Y, Yao X, Zhang B, DU X. Comparison of recovery effect for sufentanil and remifentanil anesthesia with $\mathrm{TCl}$ in laparoscopic radical resection during colorectal cancer. Oncol Lett 2016; 11:3361-5

21. Ech Y, Nikiteas N, Perrea D, Tsigris C. Effect of laparoscopic surgery on oxidative stress response: systematic review. Surg Laparosc Endosc Percutan Tech 2013; 23:101-8.

22. Liu X, Gu X, Yu M, Zi Y, Yu H, Wang Y, et al. Effects of ginsenoside $\mathrm{Rb} 1$ on oxidative stress injury in rat spinal cords by regulating the eNOS/Nrf2/HO-1 signaling pathway. Exp Ther Med 2018; 16:1079-86. 